\title{
LES FONCTIONS DU MENTOR DE L'ENTREPRENEUR NOVICE ${ }^{1}$
}

\author{
Etienne St-Jean, professeur \\ Département des Sciences de la Gestion \\ Institut de Recherche sur les PME \\ Université du Québec à Trois-Rivières \\ 3351, boul. des Forges, CP 500 \\ Trois-Rivières, Québec G9A 5H7 \\ Tél. : (819) 376-5011 poste 4293 \\ Fax : (819) 376-5138 \\ Courriel : etienne.st-jean@uqtr.ca
}

\section{RÉSUMÉ}

Si l'éclosion des programmes d'accompagnement dans plusieurs pays industrialisés, notamment ceux qui s'inspirent du mentorat, a permis de mieux comprendre les retombées attendues de ces interventions, les fonctions exercées par les mentors d'entrepreneurs novices ne sont pas connues à ce jour. Dans le cadre d'une recherche menée auprès du plus important programme de mentorat au Québec, le Réseau M de la Fondation de l'entrepreneurship, ces fonctions ont été investiguées et les résultats démontrent qu'elles se regroupent en trois catégories : les fonctions psychologiques, celles reliées à la carrière et celle de modèle de rôle. Un outil de mesure en neuf items est également proposé et validé, permettant alors d'évaluer le mentorat reçu par l'entrepreneur novice accompagné par un mentor.

\section{INTRODUCTION}

Depuis quelques années, on assiste à une éclosion de programmes de mentorat d'affaires conçus pour offrir du soutien aux entrepreneurs. À titre d'exemple, le programme SCORE ${ }^{2}$ aux ÉtatsUnis, fondé dans les années 1970 et financé par la Small Business Administration, a soutenu plus de 8 millions de dirigeants de petites entreprises grâce à son réseau de plus de 12000 mentors bénévoles. En Europe, d'autres initiatives similaires existent telles que celle soutenue par le Business Link d'Angleterre, le programme Mentor Eget Företag de Suède ou France Initiative, en France, avec près de 5000 mentors bénévoles, pour ne nommer que ceux-là. Le mieux connu de ces programmes au Québec est celui créé en 2000 par la Fondation de l'entrepreneurship (www.entrepreneurship.qc.ca), un organisme voué à la promotion de la culture entrepreneuriale. Maintenant connu sous le nom de Réseau $M$, ce programme qui a vu le jour à l'aube de l'an 2000 offre un service d'accompagnement aux entrepreneurs novices par le biais d'environs 70 cellules de mentorat dispersées sur l'ensemble du territoire du Québec. En 2010, selon les estimations de la Fondation, plus de 3500 entrepreneurs novices avaient bénéficié du soutien de l'un des 1200 mentors accrédités par les organismes collaborateurs.

Certains travaux laissent entendre que les entrepreneurs novices qui sont soutenus par des mentors pourraient bénéficier de plusieurs types de retombées, notamment des apprentissages cognitifs (nouvelles connaissances et compétences, amélioration de la vision d'affaires et identification d'opportunités), des apprentissages affectifs (réduction de la solitude, augmentation de l'auto-efficacité et image de soi), de nouveaux contacts et parfois même, des changements dans la PME (croissance des ventes ou meilleure rentabilité) (Bisk, 2002; Nandram, 2003; Wikholm et al., 2005; St-Jean, 2008). L'entrepreneur novice, soit celui qui a

\footnotetext{
${ }^{1}$ St-Jean, É. (2010), «Les fonctions du mentor de l'entrepreneur novice », Revue de l'entrepreneuriat, vol. 9 , no. 2, p. 28-49.

${ }^{2}$ Acronym for Service Corps of Retired Executives. Info at www.score.org.
} 
démarré un projet d'affaires et qui n'a pas d'expérience préalable comme entrepreneur, bénéficie alors des schémas cognitifs des entrepreneurs d'expérience, ce qui stimule alors leurs apprentissages (Baron et Ensley, 2006). Bien que l'on connaisse maintenant un peu mieux les retombées pour le novice, les fonctions exercées par le mentor pour permettre leur développement ne sont que très peu connues à ce jour.

Pourtant, dans la documentation scientifique s'intéressant au mentorat dans d'autres contextes, notamment dans la grande organisation, où un employé identifié comme ayant un certain potentiel (le protégé ${ }^{1}$ ) est jumelé à un autre en position hiérarchique (le mentor), les rôles du mentor ont été explorés à de nombreuses occasions (Kram, 1985; Fowler, 2002; Levesque et al., 2005). Ces fonctions du mentor constituent même une mesure du mentorat reçu par le protégé. Sachant l'importance que revêt l'accompagnement de type «mentorat » chez les entrepreneurs novices, il devient primordial de mieux comprendre en quoi consiste l'intervention d'un mentor pour permettre le développement de ces retombées. En outre, même si cette recherche ne vise pas à évaluer si le mentorat s'adresse à une catégorie d'entrepreneur différente des autres (peutêtre que le mentorat n'est pas utile pour certains davantage autonomes), elle permet au moins de d'entrer à l'intérieur d'une intervention et de documenter le « comment » du mentorat.

Le présent travail tentera donc de combler cette lacune, en documentant les fonctions du mentor de l'entrepreneur dans le contexte du réseau de mentorat d'affaires de la Fondation de l'entrepreneurship. Pour y parvenir, la documentation scientifique mise à contribution pour cerner le concept sera présentée en premier lieu. Puisque la documentation n'a pas fourni suffisamment de détails sur l'objet de recherche, une démarche exploratoire s'est avérée nécessaire et elle sera exposée dans un deuxième temps. Par la suite, l'ensemble de la démarche confirmatoire, qui a permis de prouver la validité empirique des fonctions du mentor, sera introduite, avant de proposer une proposition parcimonieuse et de discuter des résultats, en concluant ce travail avec des pistes pour les recherches futures.

\section{ANALYSE DE LA DOCUMENTATION PERTINENTE}

Dans cette section, nous présenterons d'abord le concept de fonctions du mentor, tel qu'il a été développé dans la littérature sur le mentorat en grande organisation. Ensuite, nous viendrons conclure la section en abordant les fonctions du mentor dans un contexte d'entrepreneur, en précisant en quoi une démarche inductive est nécessaire préalablement à une démarche déductive.

\subsection{LES FONCTIONS DU MENTOR DANS LA GRANDE ORGANISATION}

Kram (1985) suggère que le mentor exerce deux fonctions principales envers son protégé : une reliée à la carrière et une autre psychologique. Dans la première, les composantes de la fonction concernent tout ce qui touche l'avancement de la carrière, soit le parrainage, la publicisation/visibilité, le coaching, la protection et l'attribution de défis. Dans l'autre fonction, celle psychologique, on retrouve ce qui se rapporte au développement d'un sentiment de compétence et de confiance en soi, soit le modèle de rôle, l'acceptation/confirmation, le conseil et l'amitié. Plusieurs recherches ont utilisé ces fonctions avec un certain succès (voir par exemple Noe (1988) ou Allen et Eby (2004)). Elles ont été testées à plusieurs reprises et on

\footnotetext{
${ }^{1}$ Le terme « protégé » est utilisé dans la littérature traitant notamment du mentorat dans la grande organisation, faisant référence au sponsorship mentoring. Lorsqu'il est question du mentorat pour un entrepreneur, le terme utilisé par les praticiens est «mentoré ». Il s'avère plus adéquat pour les entrepreneurs que " protégé » ou même " parrainé » puisque ceux-ci apprécient moins l'évocation au besoin de protection, ce qui n'est pas le cas lorsque l'on utilise «mentoré ». Ce sera ce terme qui sera utilisé ici lorsqu'on fait référence au mentorat d'un entrepreneur.
} 
remarque une invariance de ces facteurs entre un groupe de femmes et un groupe d'hommes, ce qui confirme que le modèle des fonctions du mentor à deux principales fonctions possède la même signification pour les deux sexes (Tepper et al., 1996).

Toujours en utilisant les travaux de Kram (1985) concernant les fonctions du mentor, Scandura (1992) a procédé à une analyse factorielle exploratoire et observe que l'item relié au modèle de rôle dans la fonction psychologique de Kram est une fonction distincte de la fonction psychologique et de celle reliée à la carrière. D'autres recherches confirment le caractère distinct de la fonction de modèle et proposent trois principales fonctions du mentor (voir par exemple Scandura et Ragins (1993), Scandura et Williams (2001), Pellegrini et Scandura (2005) ou Bouquillon et al. (2005)).

Il n'est pas étonnant de constater que la plupart des recherches trouvent deux ou trois fonctions en lien avec les items proposées par Kram (1985) puisque $42 \%$ des études sur le mentorat se basent sur les travaux de celle-ci (Hansford et al., 2002). Selon nous, cette situation empêche l'élaboration de théories concurrentes qui pourraient nuancer les découvertes sur le mentorat, notamment au niveau des fonctions que joue le mentor. D'ailleurs, dans une recherche exploratoire traitant des comportements du mentor dans l'organisation, Levesque et al. (2005) interrogent des protégés à ce sujet et leur demandent de classer les comportements du mentor selon l'ordre d'importance à leurs yeux. Ils constatent que le coaching ressort comme principal comportement souhaité, lequel est suivi du soutien informationnel, de la visibilité, du soutien politique, de la "publicisation », du modèle de rôle, de la motivation, de la formation, de la protection, de la proposition de défis, du conseil personnel, de l'amitié, du soutien matériel, de la relativisation des problèmes, de l'acceptation/confirmation et enfin du soutien à propos du statut. Comme le font remarquer les auteurs, les protégés vont juger le soutien informationnel comme étant un comportement très important ${ }^{1}$ de leur mentor. Pourtant, les fonctions du mentor de Kram (1985) ne font même pas mention de cet aspect (Levesque et al., 2005: p. 438). Tout porte à croire que les items de Kram ne sont peut-être pas complets et qu'une démarche inductive est encore souhaitable à propos de ce concept, même dans le domaine du mentorat organisationnel.

En outre, toujours en utilisant une démarche inductive, Fowler (2002) obtient sept fonctions identifiées par les protégés, lesquelles sont différentes des fonctions de Kram, et lorsqu'elle interroge les mentors, elle en observe huit distinctes. Les protégés mentionnent que le mentor, dans un contexte organisationnel, offre un rôle de guide personnel et émotionnel, une facilitation à l'apprentissage, de la remise en question, un modèle de rôle, de la facilitation dans le développement de la carrière, des conseils relatifs aux systèmes et stratégies ainsi que de l'amitié. Bref, l'auteure démontre que le travail de Kram n'est sans doute plus actuel, puisqu'il faut considérer que les organisations changent, ainsi que les besoins des protégés, ce qui fait évoluer le soutien qu'offre le mentor dans l'organisation.

D'autres recherches vont proposer des fonctions ou des rôles que le mentor joue dans d'autres contextes que celui dans la grande organisation. Par exemple, suite à une recherche inductive visant le développement d'un outil de mesure des fonctions du mentor dans le contexte de l'enseignement post-secondaire, Cohen (1995) suggère que le mentor possède six différentes fonctions. La première concerne «l'emphase sur la relation », soit la capacité du mentor à créer un climat psychologique de confiance permettant au protégé de partager ses expériences personnelles. La deuxième est «l'emphase sur l'information» et se rapporte à la capacité du mentor de demander des informations détaillées afin d'offrir des suggestions spécifiques au protégé. La troisième fonction est la « facilitation de la focalisation » qui permet au mentor de

\footnotetext{
$146 \%$ des répondants considèrent le soutien informationnel parmi les trois plus importants comportements du mentor organisationnel.
} 
guider le protégé dans une exploration et une recension de ses intérêts, habiletés, idées et croyances en lien avec sa carrière ou sa formation. Vient ensuite la fonction de « confrontation de la focalisation » qui remet en question, de manière respectueuse, les décisions ou les évitements du protégé dans son développement personnel. L'avant-dernière fonction est celle du «modèle » lorsque le mentor partage ses expériences et ses sentiments appropriés pour permettre de personnaliser et d'enrichir la relation. Finalement, la dernière fonction est celle de la "vision du protégé » que le mentor doit stimuler dans un contexte de pensée critique, lui permettant de développer sa capacité à fonctionner de façon indépendante pour atteindre ses objectifs personnels. L'auteur fournit peu de détails concernant l'élaboration de ces fonctions, se référant à son travail doctoral. Néanmoins, selon quelques statistiques fournies par celui-ci, tout porte à croire que cet outil mesure six composantes distinctes, lesquelles mesurent ensemble les fonctions du mentor envers son protégé.

Dans une proposition conceptuelle, Houde (1995: p. 106-107) suggère l'existence de douze différentes fonctions que peut jouer un mentor, lesquelles ne se rattachent pas exclusivement à un contexte organisationnel mais plutôt au mentorat de façon générale :

1. Accueillir le protégé dans le milieu, le présenter aux autres membres

2. Guider le protégé dans le milieu en lui faisant part des normes, des valeurs et des tabous de la culture organisationnelle

3. Enseigner au protégé

4. Entraîner le protégé à acquérir des habiletés précises reliées à la pratique d'un travail

5. Répondre du protégé auprès des autres membres du milieu : le mentor est pour ainsi dire le répondant du protégé

6. Favoriser l'avancement du protégé dans ce milieu

7. Être le modèle du protégé : le protégé a besoin de s'identifier à son mentor (avant de s'en différencier)

8. Présenter des défis au protégé et lui fournir l'occasion de faire ses preuves

9. Conseiller le protégé sur une question ou l'autre

10. Donner de la rétroaction directe, utile et constructive

11. Soutenir moralement le protégé, particulièrement en période de stress

12. Sécuriser le protégé

Selon l'auteure, ces fonctions peuvent s'exercer en visant le développement professionnel de l'individu ou en favorisant le développement intégral de la personne, parfois l'un au travers l'autre mais peut-être aussi l'un sans l'autre.

Dans un examen de la documentation sur les différentes formes de mentorat, Jacobi (1991) recense huit auteurs qui ont documenté les fonctions du mentor, regroupant au total quinze différentes fonctions. Elle note l'acceptation/le soutien/l'encouragement, le conseil/la guidance, l'accès aux ressources/outrepasser la bureaucratie, la proposition de défis/d'opportunités, la clarification des valeurs et des buts, le coaching, l'information, la protection, le modèle de rôle, le statut social, la socialisation/guide et hôte, la défense/commandite, la stimulation de l'acquisition de savoirs, la formation/l'instruction et la visibilité/l'exposition. Dans le contexte plus large des relations de type « développementales », c'est-à-dire qui visent le développement personnel autant que professionnel, incluant généralement le mentorat mais également d'autres formes d'accompagnement (Rock et Garavan, 2006), McCauley et Douglas (2004) proposent différents rôles que jouent les développeurs, tout comme D'abate et al. (2003) qui indiquent que les comportements des développeurs (que l'on considère généralement comme des mentors) peuvent se regrouper en trois principaux rôles : apprentissage, soutien émotionnel et progression à la carrière. Ces rôles se divisent à leur tour en 23 différentes composantes. On peut donc constater qu'autant dans le contexte organisationnel que dans la perspective de relations développementales, le mentor peut exercer différentes fonctions. 


\subsection{LES FONCTIONS DU MENTOR DANS UN CONTEXTE D'ENTREPRENEUR}

À ma connaissance, la recherche de Waters et al. (2000) est la seule où il a été testé de façon empirique un outil de mesure des fonctions du mentor de l'entrepreneur. En se basant sur les travaux de Kram (1985), ils arrivent à une «construction" semblable à elle, laquelle possède deux fonctions : l'une reliée à la «carrière" et l'autre psychologique. Avant d'utiliser cette recherche comme un outil de mesure dans un contexte entrepreneurial, il convient de l'analyser plus en détail. D'abord, malgré le fait que Waters et al. (2000) reconnaissent que les fonctions du mentor organisationnel de Kram ne peuvent pas tout à fait s'appliquer dans un contexte entrepreneurial, ils indiquent que leur outil se base sur la littérature du mentorat tel qu'il se pratique dans la grande organisation. L'adaptation des fonctions du mentor « organisationnel» dans un contexte entrepreneurial a de fortes chances de rendre l'outil développé incomplet, voire même inapproprié. De plus, le contexte de l'étude vient largement teinter le développement de l'outil des auteurs. Au-delà du fait que le mentor de leur étude pourrait jouer davantage le rôle d'un coach, lequel "agit comme un guide dans l'implantation du plan d'affaires de son protégé ", on peut constater que les items sélectionnées pour développer le construit se basent sur les éléments même du programme dans lequel s'insère les relations observées. Ainsi, leurs items comprennent les éléments en lien direct avec le programme, soit le fait de fournir de l'assistance technique, de l'assistance marketing, des conseils au niveau de la finance et au niveau légal. Les auteurs ajoutent deux items plus généraux, soit le réseautage et la réponse aux besoins de « bureau ». Lorsqu'ils testent l'outil auprès de leur échantillon, seul les items reliés aux objectifs du programme vont contribuer à la validité de la mesure, obligeant les chercheurs à retrancher les items généraux qu'ils ont ajoutés. Selon nous, il ne fait pas de doute que le contexte particulier de l'étude rende impossible la généralisation de l'outil et son utilisation auprès d'un autre programme de mentorat. Qui plus est, malgré le fait que Waters et al. (2000) se basent sur les recherches du mentorat dans le contexte de la grande organisation pour soutenir le développement de leur outil dans un contexte entrepreneurial, ils font complètement abstraction d'une fonction très importante du mentor, la fameuse "troisième " fonction du mentor, soit celle de modèle de rôle. Même si cette facette du rôle du mentor fait l'objet d'un grand consensus chez les chercheurs dans le domaine organisationnel et que théoriquement, le mentor entrepreneur pourrait fort bien jouer cette fonction auprès de son protégé, comme le rapportent Wanberg et al. (2003), Waters et al. (2000) n'en font même pas mention. Pourtant, on indique que les gens dans l'entourage de l'entrepreneur servent de modèle à ceux-ci et pourraient influencer leur volonté d'être en affaires (Diochon et al., 2001; Gasse, 2003; Arenius et Minniti, 2005). Cela laisse présager qu'en plus du caractère très contextuel de l'outil, il est en outre très probable qu'il soit incomplet.

Une récente étude réalisée auprès des mentors d'entrepreneurs novices de la Fondation de l'entrepreneurship révèle que ceux-ci ont de la difficulté à définir leurs rôles considérant l'étendue des besoins exprimés par les protégés (Fortin et Simard, 2007). D'autres recherches réalisées dans un contexte de mentorat de l'entrepreneur laissent présager certaines fonctions du mentor. Par exemple, le mentor peut aider l'entrepreneur à identifier les habiletés et les savoirs critiques à sa réussite, même dans un contexte d'entrepreneuriat d'équipe (Shepherd et Krueger, 2002). Dans le cadre d'un groupe d'entrepreneurs formés pour soutenir le processus de démarrage des participants, il est apparu que le groupe jouait particulièrement cinq rôles : la transformation du rêve à la réalité par le soutien du processus de développement de la «vision d'affaires ", l'augmentation des aspirations, la stimulation des idées et de la créativité, l'aide pratique/le soutien concret face aux problèmes rencontrés et le soutien psychologique qui réduit notamment la solitude du dirigeant (Rush et al., 1987). Certains mentors d'entrepreneurs novices vont leur fournir de l'encouragement, en jouant un rôle de motivateur, et vont aussi servir de modèle tout en donnant de la rétroaction, des conseils et des contacts d'affaires (McGregor et Tweed, 2002). Le rôle des mentors pour fournir des contacts à leur mentoré est aussi observé par d'autres auteurs, tout comme le modèle de rôle (Couteret et al., 2006). Malgré 
ces quelques constats, aucun consensus ne semble émerger de la documentation scientifique. Cela suggère de procéder de manière exploratoire et inductive, dans un premier temps, avant de pouvoir tester un outil de mesure des fonctions.

\section{MÉTHOdOLOGIE}

L'objectif de cette partie est de répondre à la question de recherche suivante : Quelles sont les différentes fonctions du mentor de l'entrepreneur? Pour y répondre, nous avons eu recours aux témoignages des participants au programme de mentorat de la Fondation de l'entrepreneurship et aux réponses de mentorés par questionnaire internet. Avant de présenter la méthode utilisée, le programme étudié sera exposé, soit le réseau de mentorat de la Fondation de l'entrepreneurship. Par la suite, les résultats de la démarche qualitative et inductive seront présentés brièvement, avant de poursuivre vers les analyses déductives sur cet objet de recherche.

\subsection{LE PROGRAMME ÉTUDIÉ}

Le réseau de mentorat d'affaires qui sert de toile de fond à la présente recherche. Le service de mentorat est offert aux entrepreneurs novices par le biais d'environs 70 cellules de mentorat dispersées sur l'ensemble du territoire du Québec. Tel que mentionné en introduction, ces cellules sont généralement soutenues par des organismes de développement économique tels les Centres locaux de développement (CLD), les Sociétés d'aide au développement des collectivités (SADC) et les chambres de commerce locales. Ces organismes assurent le développement du programme au niveau local ou régional, tout en adhérant au modèle du mentorat d'affaires développé par la Fondation. De façon plus précise, les organismes locaux se dotent d'un coordonnateur de la cellule, lequel est responsable du recrutement des mentors, de l'organisation des séances de formation à leur intention, de la promotion du programme auprès des entrepreneurs novices, du jumelage des participants et de la supervision de la relation qui s'ensuit. Les entrepreneurs novices peuvent bénéficier du soutien d'un mentor pour un coût minime, soit quelques centaines de dollars annuellement, et parfois même gratuitement. Afin de bien encadrer le développement local, la Fondation de l'entrepreneurship développe des ateliers de perfectionnement orientés sur la relation mentor-mentoré pour éclairer concrètement le rôle des mentors auprès des nouveaux entrepreneurs. Basé sur un code d'éthique d'intervention où la confidentialité des relations est d'une importance capitale, le service de mentorat d'affaires s'est également doté d'un contrat-type pour encadrer et guider les parties dans le choix des modalités de leur relation et des objectifs à atteindre. Il s'agit donc d'un mentorat de type « formel ».

Il faut aussi souligner que les entrepreneurs novices ne sont pas obligés de recourir au service, par exemple en échange de l'obtention d'un prêt. Ils le font sur une base volontaire et intéressée. Tous les mentors du réseau sont des bénévoles. Leur objectif principal est de contribuer au développement d'un nouvel entrepreneur, une manière de redonner un peu de ce qu'ils ont reçu parfois eux-mêmes, de manière informelle toutefois. Bien que certains mentors possèdent certaines spécialités, ils ne sont pas recrutés pour leurs compétences précises. D'abord et avant tout, on exige d'eux qu'ils soient en mesure de bien écouter leur mentoré et de l'aider à trouver lui-même les réponses à ses questions. Il s'agit d'une démarche basée sur l'apprentissage de l'entrepreneur novice, pour l'aider à « faire du sens » de ses propres expériences.

\subsection{ANALYSE INDUCTIVE}

Les différentes fonctions qu'exerce le mentor ont été explorées en 2005 et 2007 par le biais de groupes de discussion de mentorés et de mentors. D'abord, en 2005, des données ont été recueillies lors de groupes de discussion réalisés dans le cadre de l'évaluation du programme de mentorat de la Fondation. Une firme spécialisée avait alors eu le mandat d'organiser ces rencontres et 40 entrepreneurs novices y ont participé. Ensuite, en 2007, deux groupes 
comprenant au total 11 mentorés ont été organisés, l'un à Montréal et l'autre à Québec. Les participants ont été sélectionnés au hasard à partir d'une liste de plus de 1000 participants du réseau de mentorat de la Fondation de l'entrepreneurship. Lors de la rencontre, il leur a été demandé de discuter des différents rôles joués par leur mentor pendant leur relation, entre autres. De plus, un groupe de discussion de mentors cette fois-ci, qui comprenait cinq hommes et trois femmes (huit participants), tous ayant tous été en affaires par le passé, a été mobilisé pour éviter un biais causé par le point de vue unique des mentorés.

L'analyse effectuée a consisté à déterminer autant de fonctions distinctes les unes des autres, de manière inductive, et à proposer des items pour une mesure subséquente, en s'assurant ainsi qu'ils correspondent aux commentaires recueillis, autant auprès des mentors que des mentorés. Une fois cet exercice complété, la proposition a été soumise à un expert académique pour en obtenir des commentaires. Des modifications ont alors été apportées. Dans un deuxième temps, la proposition modifiée a été soumise à un groupe d'experts en mentorat, composé du directeuradjoint au service de mentorat d'affaires de la Fondation de l'entrepreneurship, d'un professeur d'université retraité, spécialisé en pédagogie et en entrepreneuriat et mentor dans le programme de la Fondation, ainsi que d'un consultant et formateur pour les mentors de la Fondation de l'entrepreneurship, lui aussi mentor dans ce même programme. Aucune de ces personnes n'ont participé aux groupes de discussion. Dans un premier temps, les experts ont reçu la liste des fonctions du mentor avec une courte définition de chacune d'elles ainsi que l'ensemble des items servant à les mesurer, mélangés de manière aléatoire. Ils devaient tenter de classer les items, selon les définitions des fonctions du mentor, et retourner le formulaire. Dans un deuxième temps, la proposition «théorique» corrigée leur a été envoyée et ils pouvaient formuler des commentaires visant à améliorer la formulation des items, à suggérer l'ajout ou le retranchement des fonctions, et ainsi de suite. Cette méthode, qui vise à améliorer la validité du contenu, s'inspire fortement des différentes méthodes recensées dans les écrits par Hinkin (1998). La possibilité que les experts puissent formuler des commentaires sur la nature des fonctions semblait une opportunité intéressante du fait que deux des trois sont mentors et sont fortement conscientisés par les rôles joués par ceux-ci. Les résultats obtenus font états de quatre fonctions de nature psychologique, quatre fonctions reliées à la carrière et une fonction de modèle de rôle (voir Tableau 1). Une fois en possession d'une proposition basée sur une démarche inductive, elle a ensuite été testée de manière déductive.

\subsubsection{Population et échantillonnage.}

La population étudiée représente l'ensemble des entrepreneurs mentorés du réseau de mentorat d'affaires de la Fondation de l'entrepreneurship, soit ceux inscrits sur la liste dressée par la Fondation à la fin d'avril 2008. L'équipe de mentorat de la Fondation s'est assurée de récupérer une adresse de courriel pour tous les mentorés de la liste, cela représentant 1545 entrepreneurs novices. Un questionnaire en ligne a été soumis aux mentorés actuellement en relation ou dont la relation est terminée mais, dans ce dernier cas, elle devait s'être déroulée pendant au moins trois rencontres. Deux rappels ont été envoyés aux non-répondants et suite à cette démarche, 158 personnes ont indiqué ne pas avoir reçu suffisamment de mentorat pour être éligible, 388 adresses de courriel se sont avérées inexactes ou abandonnées par les utilisateurs et 18 ont signalé une erreur de liste. En somme, sur les 981 courriels valides, 362 mentorés ont répondu au questionnaire, pour un taux de réponse de $36,9 \%$, procurant une marge d'erreur de 4,4\%, 19 fois sur 20. J'ai testé le biais du non-répondant en suivant la procédure d'Armstrong et Overton (1977) et aucune variables démographiques ou d'intérêt est significativement différente entre les répondants tardifs et les hâtifs, ce qui permet de présumer à la représentativité de l'échantillon. 


\section{Tableau 1}

Les neuf fonctions du mentor de l'entrepreneur

\begin{tabular}{|c|c|}
\hline \multicolumn{2}{|r|}{ Fonctions psychologiques } \\
\hline Réflecteur & $\begin{array}{l}\text { Le mentor permet au mentoré d'avoir de la rétroaction sur ce qu'il est et sur son projet } \\
\text { d'entreprise. Il lui reflète l'image qu'il projette aux autres, un peu à la manière d'un } \\
\text { miroir. Cette fonction fournit au mentoré une sorte de bilan personnel, dans une } \\
\text { perspective d'identification des forces sur lesquelles miser et des faiblesses à travailler. }\end{array}$ \\
\hline Sécurisation & $\begin{array}{l}\text { Le mentor sécurise le mentoré dans les périodes difficiles. Il sert de soupape pour } \\
\text { évacuer le stress accumulé et pour lui permettre de relativiser ses problèmes. }\end{array}$ \\
\hline Motivation & $\begin{array}{l}\text { Le mentor motive et encourage le mentoré. Il lui fait sentir qu'il a confiance en ses } \\
\text { capacités et l'incite à persévérer. }\end{array}$ \\
\hline Confident & $\begin{array}{l}\text { Au fil du temps, le mentoré peut se confier comme il le ferait auprès d'un ami. La } \\
\text { relation entre le mentor et son mentoré se transforme aussi parfois en amitié. }\end{array}$ \\
\hline \multicolumn{2}{|r|}{ Fonctions reliées à la carrière } \\
\hline Intégration & $\begin{array}{l}\text { Le mentor facilite l'intégration du mentoré dans la communauté d'affaires en le } \\
\text { présentant à des personnes qui peuvent l'aider lorsqu'il en a besoin. }\end{array}$ \\
\hline $\begin{array}{l}\text { Soutien } \\
\text { informationnel }\end{array}$ & $\begin{array}{l}\text { Le mentor donne de l'information au mentoré. Il lui transfert des connaissances } \\
\text { personnelles de diverses natures, par exemple au niveau de la gestion d'une entreprise, } \\
\text { des lois en vigueurs, des informations utiles au secteur d'activité, et ainsi de suite. }\end{array}$ \\
\hline Confrontation & $\begin{array}{l}\text { Le mentor confronte les idées du mentoré pour lui permettre d'aller plus loin dans sa } \\
\text { réflexion. Cette confrontation se déroule dans le contexte de la résolution d'un problème } \\
\text { dans lequel le mentoré ne fait pas partie de la solution mais du problème lui-même, à } \\
\text { cause de croyances, d'attitudes ou d'habitudes qui l'empêchent d'avancer vers ses } \\
\text { objectifs. }\end{array}$ \\
\hline Guide & $\begin{array}{l}\text { Dans le contexte de la résolution d'un problème, le mentor aide le mentoré à améliorer } \\
\text { sa compréhension du problème et à élargir sa vision du problème et de son contexte. } \\
\text { Lorsque cela s'avère nécessaire, le mentor lui fait part aussi de ses suggestions et de ses } \\
\text { conseils pour le résoudre. }\end{array}$ \\
\hline \multicolumn{2}{|r|}{ Fonction de modèle de rôle } \\
\hline Modèle & $\begin{array}{l}\text { La fonction de modèle concerne le mentor pour ce qu'il est en tant que personne. Lors } \\
\text { des rencontres, le mentor expose des épisodes de sa vie et le mentoré tire alors les leçons } \\
\text { qui s'imposent, en fonction de sa situation personnelle. Le mentor est aussi une personne } \\
\text { qui peut être une source d'inspiration ou, à tout le moins, de comparaison. }\end{array}$ \\
\hline
\end{tabular}

Au niveau des caractéristiques des mentorés, l'échantillon contient 162 hommes $(51,6 \%)$ et 152 femmes $(48,4 \%)$, cela représentant presque la parité dans les sexes. Les jumelages ont été faits auprès de 275 mentors hommes $(81,4 \%)$ et de seulement 63 mentors femmes $(18,6 \%)$. Cette situation est cependant «normale », en considérant la plus grande représentation des hommes parmi les mentors disponibles. Mentionnons aussi que la plupart des mentors sont des entrepreneurs de carrière $(47,9 \%)$ mais qu'une assez grande proportion ont été (ou sont toujours) cadres dans l'entreprise privée $(34,3 \%)$. Un faible nombre d'entre eux ont été fonctionnaires $(6,8 \%)$ et certains mentorés ignoraient la carrière de leur mentor $(10,9 \%)$. Au moment du jumelage, certains mentors étaient toujours professionnellement actifs $(40,8 \%)$ alors qu'une majorité étaient à la retraite $(57,4 \%)$. La vaste majorité $(79,6 \%)$ des mentors n'ont pas évolué dans le même secteur d'activité que leur mentoré, conformément à ce que suggèrent les dirigeants du réseau à la Fondation, ce qui évite les conflits d'intérêt potentiel et assure que le mentor demeure un généraliste plutôt qu'un spécialiste technique.

L'âge moyen des mentorés est de 39,81 ans (écart-type de 8,97, médiane de 38 ans) et varie entre 23 et 70 ans. Ils sont assez scolarisés, $55 \%$ d'entres-eux possèdent au moins un diplôme universitaire. La presque totalité des mentorés avaient une entreprise active au moment du jumelage (293 sur 314, 93,3\%) et les autres étaient en processus de démarrage. Celle-ci avait 
peu d'employés, avec une moyenne de 4,48 (écart-type 9,69, médiane 2). Le chiffre d'affaires est majoritairement inférieur à $100000 \$$ annuellement $(62,8 \%), 88,9 \%$ ayant moins de $500000 \$$ de c.a. par an et pour seulement $8,6 \%$, il était supérieur à 1 million $\$$. Au niveau des profits bruts, incluant les salaires et bonis du dirigeant principal, la situation n'est guère meilleure. La très grande majorité $(68,1 \%)$ déclarent avoir des profits inférieurs à $25000 \$$ annuellement, $83,5 \%$ font moins de $50000 \$$ et seulement $6,3 \%$ font plus de $100000 \$$. Les secteurs industriels sont variés, mais avec une légère concentration dans les services professionnels $(23,0 \%)$, dans la fabrication $(14,4 \%)$ et dans le commerce de détails $(11,9 \%)$. Les relations de mentorat ont duré en moyenne 16,07 mois (écart-type de 14,4, médiane de 13), les rencontres avec le mentor duraient en moyenne 68,52 minutes (écart-type de 14,4, médiane de 67) et il y avait un peu moins d'une rencontre par mois $(0,807)$, la médiane étant d'une rencontre par mois.

\subsubsection{Méthode}

Pour vérifier la validité de l'outil, il s'avère essentiel de considérer l'unidimensionnalité de chacune des sous-fonctions du mentor. Un ensemble d'items ne doit référer qu'à un seul construit pour qu'il soit considéré valide (Hattie, 1985). À cet effet, on indique que l'analyse factorielle confirmatoire s'avère supérieure à d'autres méthodes pour évaluer l'unidimensionnalité des construits (Gerbing et Anderson, 1988). On suggère aussi que les indices de consistance interne soient divulgués lors de la création ou de l'utilisation de variables latentes (Shook et al., 2004; Slater et Atuahene-Gima, 2004). Conséquemment, une analyse factorielle confirmatoire ainsi que des analyses de consistance interne seront effectuées.

Pour s'assurer de la fiabilité de l'instrument de mesure, on suggère la méthode du « test-retest » et la méthode des formes alternatives (Drucker-Godard et al., 2003). La première consiste à effectuer le même test sur les mêmes individus à des périodes différentes. La seconde suggère d'administrer deux tests aux mêmes individus, le second test n'étant pas identique et constitue une forme alternative supposée mesurer le même phénomène. Contrairement à la méthode «test-retest », cette dernière méthode permet de réduire l'effet de mémoire ${ }^{1}$. Pour cette recherche, 173 répondants ont répondu à la fois à la première version du questionnaire, où l'ensemble des 35 items représentant les 9 sous-fonctions étaient mélangés, ainsi qu'au deuxième questionnaire, où les 9 sous-fonctions étaient explicitées et les items classés en conséquence. Il ne s'agit donc pas exactement d'une application de la méthode «test-retest» puisqu'il y a eu des modifications dans la présentation, mais elle peut s'en approcher. Il ne s'agit pas non plus d'une application de forme alternative, puisque les mêmes items ont été administrés, mais ceux-ci l'ont été dans un ordre différent. Bref, il s'agit d'une voie alternative. Néanmoins, pour permettre de juger de la fiabilité de l'outil, les corrélations entre chacune des sous-fonctions initialement répondues et celles reprises par les répondants seront présentées. Il faut noter que le délai entre l'administration du questionnaire initial et celui révisé varie selon les répondants, certains ayant pu répondre à la version révisée le lendemain de la version initiale et d'autres près de six (6) semaines plus tard. Pour des raisons logicielles ${ }^{2}$, ce délai n'a pas pu être calculé.

Finalement, pour s'assurer de la validité du construit, il a été demandé aux répondants d'indiquer à combien de personnes le mentor les a présentés. La fonction d'intégration, qui consiste justement à vérifier dans quelle mesure le mentor a joué son rôle en le présentant à d'autres personnes, devrait être corrélée plus fortement avec le nombre de personnes effectivement présentées. Cette analyse sera donc effectuée. En outre, il est possible de penser que les mentors qui ont été entrepreneurs eux-mêmes dans leur carrière pourraient déployer

\footnotetext{
${ }^{1}$ Les répondants pourraient se souvenir de leurs réponses précédentes.

${ }^{2}$ Le logiciel de www.surveymonkey.com ne permet pas de faire des liens entre un même répondant ayant été sollicité pour deux questionnaires différents. Les réponses ont été jumelées manuellement mais la date de réponse n'a pas pu être comptabilisée.
} 
davantage la fonction de modèle de rôle. Cette vérification sera présentée également. Pour chaque fonction, une analyse factorielle confirmatoire a été réalisée à l'aide du logiciel Lisrel. Puisque les variables utilisées sont catégoriques mais ordonnées ${ }^{1}$, il s'avère judicieux d'utiliser le logiciel PRELIS puisqu'il permet de calculer une matrice de corrélation polychorique. Cette matrice s'avère supérieure pour réduire les biais d'estimation, d'autant plus qu'elle n'est pas sensible à la forme que prend la distribution marginale (Jöreskog et Sörbom, 2002; Tabachnick et Fidell, 2007). Les équations structurelles ont été réalisées avec ce type de matrice. Tel que déjà mentionné, les non-répondants à au moins un item ont été retirés de l'analyse, cette procédure permettant d'améliorer la qualité de la matrice (Jöreskog et Sörbom, 2002). Au total, 159 répondants ont donc été utilisés pour l'analyse. Pour les autres analyses, les corrélations de Pearson ont été calculées.

\section{RÉSULTATS OBTENUS}

Les résultats des analyses de validation des fonctions seront présentés, en abordant d'abord les fonctions psychologiques, pour ensuite faire état des fonctions reliées à la carrière et de la fonction de modèle de rôle. Certains résultats de tests de validations seront inclus dans les résultats, avant de conclure cette section avec une proposition d'outil de mesure parcimonieux des fonctions du mentor.

\subsection{LES FONCTIONS PSYCHOLOGIQUES}

L'analyse inductive auprès des groupes de discussion révélait que les fonctions psychologiques semblaient correspondre à quatre sous-fonctions : la fonction de réflecteur (4 items suggérés), la fonction de sécurisation ( 3 items suggérés), la fonction de motivation (4 items suggérés) et la fonction de confident (4 items suggérés). Le Tableau 2 présente les moyennes, écarts-type et corrélations entre les différents items des fonctions psychologiques. Le modèle a été testé de manière à ce que la fonction psychologique soit reflétée par les quatre sous-fonctions, lesquelles sont à leur tour reflétées par les items créés dans la section précédente. Toutes les relations entre les variables manifestes et latentes sont significatives à $p \leq 0,01$. On constate aussi que la plupart des coefficients d'erreurs des variables manifestes sont bas, la majorité n'étant pas significatifs à $p \leq 0,05$.

Le modèle proposé possède un $\chi^{2}$ de 151,71 pour 86 degrés de liberté $(p \leq 0,0000)$, un RMSEA de 0,06954 , un SRMR de 0,03978 et un CFI de 0,9919. Le modèle est donc jugé très acceptable et aucune modification ne s'impose. L'analyse de l'alpha Cronbach $(\alpha)$ révèle un résultat de 0,889 pour la fonction de Réflecteur, de 0,916 pour la fonction de Sécurisation, de 0,953 pour la fonction de Motivation et de 0,931 pour la fonction de Confident. Les résultats obtenus par le premier questionnaire (items mélangés) et le second (items classés) ont été comparés afin de vérifier la fidélité des mesures. Pour effectuer cette analyse, les scores des mesures latentes ont été calculés dans un premier temps, pour ensuite connaître la corrélation entre ces scores provenant des deux questionnaires (le «mélangé » et le "classé »). Comme on peut le voir au Tableau 3, les corrélations entre les mesures des construits des deux collectes sont très élevées et toutes significatives à $p \leq 0,001$. Cela confirme que les mesures des fonctions psychologiques du mentor sont fiables puisque malgré la modification du questionnaire et le temps qui s'est écoulé entre les deux réponses à ces items, les construits sont fortement corrélés entre eux.

\footnotetext{
${ }^{1}$ Il s'agit de variables de type Likert à sept points.
} 
Tableau 2.Moyennes, écarts-type et corrélations entre les items des fonctions psychologiques

\begin{tabular}{llccccccccccccccc}
\hline Variable & Moy. & $\begin{array}{c}\text { E.- } \\
\text { T. }\end{array}$ & $\mathbf{1}$ & $\mathbf{2}$ & $\mathbf{3}$ & $\mathbf{4}$ & $\mathbf{5}$ & $\mathbf{6}$ & $\mathbf{7}$ & $\mathbf{8}$ & $\mathbf{9}$ & $\mathbf{1 0}$ & $\mathbf{1 1}$ & $\mathbf{1 2}$ & $\mathbf{1 3}$ & $\mathbf{1 4}$ \\
\hline 1-REF1 & 5.38 & 1.49 & & & & & & & & & & & & & & \\
2-REF2 & 5.39 & 1.49 & .88 & & & & & & & & & & & & & \\
3-REF3 & 5.08 & 1.32 & .76 & .74 & & & & & & & & & & & & \\
4-REF4 & 5.73 & 1.43 & .76 & .78 & .67 & & & & & & & & & & & \\
5-SEC1 & 5.02 & 1.58 & .66 & .66 & .57 & .66 & & & & & & & & & & \\
6-SEC2 & 5.21 & 1.58 & .71 & .69 & .62 & .68 & .90 & & & & & & & & & \\
7-SEC3 & 5.57 & 1.45 & .74 & .73 & .62 & .77 & .82 & .86 & & & & & & & & \\
8-MOT2 & 5.83 & 1.36 & .74 & .70 & .60 & .69 & .70 & .79 & .80 & & & & & & & \\
9-MOT1 & 5.98 & 1.38 & .71 & .70 & .64 & .62 & .69 & .74 & .72 & .84 & & & & & & \\
10-MOT3 & 5.98 & 1.38 & .74 & .69 & .56 & .71 & .73 & .75 & .78 & .92 & .87 & & & & & \\
11-MOT4 & 6.05 & 1.29 & .72 & .68 & .57 & .62 & .64 & .70 & .70 & .89 & .94 & .91 & & & & \\
12-CONF2 & 5.23 & 1.70 & .59 & .68 & .60 & .63 & .73 & .72 & .74 & .67 & .67 & .64 & .63 & & & \\
13-CONF3 & 4.74 & 1.82 & .54 & .60 & .59 & .50 & .64 & .69 & .61 & .60 & .64 & .55 & .58 & .82 & & \\
14-CONF1 & 5.33 & 1.69 & .60 & .69 & .57 & .64 & .73 & .70 & .73 & .63 & .71 & .63 & .67 & .89 & .77 & \\
15-CONF4 & 5.09 & 1.80 & .58 & .61 & .63 & .57 & .68 & .71 & .69 & .63 & .62 & .62 & .62 & .83 & .85 & .83 \\
\hline
\end{tabular}

Tableau 3. Corrélation entre les fonctions psychologiques du premier et du deuxième questionnaire

\begin{tabular}{ll}
\hline Fonction de réflecteur & $0,736^{* * *}$ \\
Fonction de sécurisation & $0,711^{* * *}$ \\
Fonction de motivation & $0,649 * * *$ \\
Fonction de confident & $0,801 * * *$ \\
\hline
\end{tabular}

$* p \leq 0,05 * * p \leq 0,01{ }^{* * *} p \leq 0,001$

\subsection{LES FONCTIONS RELIÉES À LA CARRIÈRE}

L'analyse inductive auprès des groupes de discussion a révélé que les fonctions reliées à la carrière consistent en quatre sous-fonctions : la fonction d'intégration (4 items suggérés), la fonction de soutien informationnel (4 items suggérés), la fonction de confrontation (4 items suggérés) et la fonction de guide (4 items suggérés). Le Tableau 4 présente les moyennes, écarts-type et corrélations des items des fonctions reliées à la carrière. Le modèle a été testé de manière à ce que la fonction reliée à la carrière soit reflétée par les quatre sous-fonctions, lesquelles sont à leur tour reflétées par les items créés dans la section précédente. Toutes les relations entre les variables manifestes et latentes sont significatives à $p \leq 0,01$. La plupart des coefficients d'erreurs des variables manifestes sont bas, la majorité n'étant pas significatifs à $p \leq 0,05$.

Concernant les indices d'adéquation du modèle, notons que le $\chi^{2}$ est de 141,20 pour 100 degrés de liberté $(p \leq 0,0042)$, un RMSEA de 0,05107, un SRMR de 0,06053 et un CFI de 0,9952. Tout comme pour les fonctions psychologiques, le modèle proposé pour les fonctions reliées à la carrière est jugé très acceptable et aucune modification ne s'impose. Au niveau de l'analyse de l'alpha Cronbach $(\alpha)$, la fonction d'intégration obtient 0,948 , celle de soutien informationnel obtient 0,899 , celle de confrontation obtient 0,882 et celle de guide obtient 0,925 . Ici aussi, on peut conclure que les résultats obtenus pour ces mesures dépassent les normes acceptables et confirment la justesse du construit relatif aux fonctions reliées à la carrière, autant du point de vue de la consistance interne que des facteurs qui le composent. 
Les résultats obtenus par le premier questionnaire et la version améliorée ont été comparés afin de vérifier la fidélité des mesures. La méthode utilisée est la même que pour les fonctions psychologiques. Comme on peut le voir au Tableau 5, les corrélations entre les mesures des construits des deux collectes sont très élevées et toutes significatives à $p \leq 0,001$. Cela confirme que les mesures des fonctions reliées à la carrière du mentor sont fiables puisque malgré la modification du questionnaire et le temps qui s'est écoulé entre les deux réponses à ces items, les construits sont fortement corrélés.

Tableau 4. Moyennes, écarts-type et corrélations entre les items des fonctions reliées à la carrière

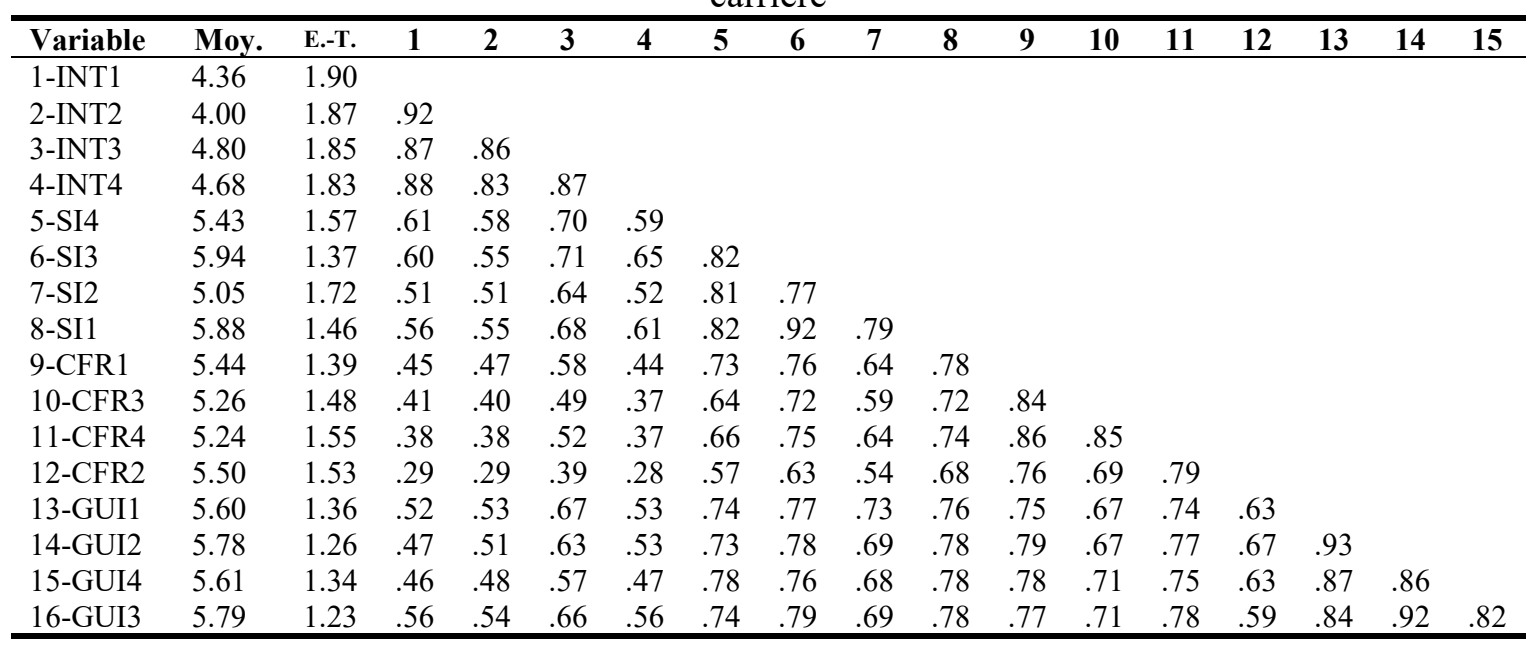

Tableau 5. Corrélations entre les fonctions reliées à la carrière du premier et du deuxième questionnaire

\begin{tabular}{lc}
\hline Fonction d'intégration & $0,772 * * *$ \\
Fonction de soutien informationnel & $0,731^{* * *}$ \\
Fonction de confrontation & $0,706^{* * *}$ \\
Fonction de guide & $0,702^{* * *}$ \\
\hline$* p \leq 0,05 * * p \leq 0,01 * * * p \leq 0,001$ & \\
& \\
On peut poser l'hypothèse que plus le mentor va déployer la fonction d'intégration, plus il \\
présentera son mentoré à d'autres personnes dans le milieu. En conséquence, on peut vérifier la \\
validité du construit en analysant la corrélation entre les fonctions reliées à la carrière et le \\
nombre de personnes présentées au mentoré. En moyenne, les mentorés déclarent avoir été \\
présentés à 3,44 personnes par leur mentor (écart-type de 3,47). Comme on peut le constater au \\
Tableau 6, la fonction d'intégration est celle qui est le plus fortement corrélée au nombre de \\
personnes présentées, ce qui confirme la validité du construit.
\end{tabular}

Tableau 6. Corrélations entre les fonctions reliées à la carrière et le nombre de personnes présentées par le mentor

\begin{tabular}{ll}
\hline Fonction d'intégration & $0,536^{* * *}$ \\
Fonction de soutien informationnel & $0,232^{* * *}$ \\
Fonction de confrontation & $0,134^{*}$ \\
Fonction de guide & $0,161 * *$ \\
\hline$* p \leq 0,05 * p \leq 0,01 * * * p \leq 0,001$ &
\end{tabular}




\subsection{LA FONCTION DE MODĖLE}

La partie inductive de la recherche a suggéré la création de 4 items pour la fonction de modèle. Le Tableau 7 présente les moyennes, écarts-type et corrélations des items de cette fonction.

Tableau 7. Moyennes, écarts-type et corrélations entre les items de la fonction de modèle

\begin{tabular}{llllll}
\hline Variable & Moy. & E.-T. & $\mathbf{1}$ & $\mathbf{2}$ & $\mathbf{3}$ \\
\hline 1-MOD1 & 5.04 & 1.66 & & & \\
2-MOD2 & 5.36 & 1.69 & .77 & & \\
3-MOD3 & 5.41 & 1.69 & .85 & .78 & \\
4-MOD4 & 5.74 & 1.52 & .76 & .93 & .76 \\
\hline
\end{tabular}

Le modèle initialement testé suggérait de laisser corréler les erreurs de mesure entre MOD1 et MOD3, ce qui a été fait. Les indices d'adéquation du modèle indiquent un $\chi^{2}$ de 0,3098 pour 1 degré de liberté $(p \leq 0,5778)$, un RMSEA de 0,00 , un SRMR de 0,002758 et un CFI de 1,000 . Avec cette modification, le modèle peut être considéré comme tout à fait acceptable. Finalement, il convient aussi de souligner que l'alpha de Cronbach est de 0,894, ce qui est aussi tout à fait acceptable. Comme pour les modèles précédents, on peut conclure que les résultats obtenus pour ces mesures dépassent les normes acceptables et confirment la justesse du construit relatif à la fonction de modèle, autant du point de vue de la consistance interne que des items qui le composent.

Qui plus est, si la fonction de modèle mesure adéquatement cette dimension, elle devrait être supérieure pour les mentorés qui ont eu des mentors entrepreneurs. En effet, la fonction de modèle de rôle comprend les items suivants : 1-Il me sert de modèle, 2-Il m'expose ses réussites et ses échecs, 3-Il est un bon exemple d'entrepreneur, et 4-Il me fait part de ses expériences d'affaires et de vie. On constate que l'item 3 concerne directement la carrière du mentor. Conséquemment, ceux ayant été en affaires devraient obtenir un meilleur score dans les réponses de leur mentoré à cet item, contrairement aux autres, qui ont été fonctionnaires, cadres dans la grande entreprise ou autre. Afin d'ajouter de la validité supplémentaire aux analyses précédentes, la différence entre les résultats des fonctions du mentor a été calculée entre les mentorés dont le mentor a été en affaires $(n=130)$ et ceux qui ne l'ont pas été $(n=139)$. Comme on peut le constater, seule la fonction de modèle est significativement différente pour les deux groupes de mentors (voir Tableau 8). Ces résultats suggèrent que la fonction de modèle mesure la dimension visée.

Tableau 8. Comparaison des fonctions du mentor selon la carrière du mentor

\begin{tabular}{lccc}
\hline \multicolumn{1}{c}{ Fonction } & $\begin{array}{c}\text { Moyenne mentor } \\
\text { «entrepreneur }\end{array}$ & $\begin{array}{c}\text { Moyenne mentor } \\
\text { «autre carrière » }\end{array}$ & $\begin{array}{c}\text { Test } t \\
\text { (sig. 2-tailed })\end{array}$ \\
\hline Fonction de réflecteur & 5,41 & 5,38 & 0,858 \\
Fonction de sécurisation & 5,34 & 5,19 & 0,394 \\
Fonction de motivation & 6,01 & 5,91 & 0,515 \\
Fonction de confident & 5,13 & 5,05 & 0,677 \\
Fonction d'intégration & 4,57 & 4,34 & 0,289 \\
Fonction de soutien inf. & 5,49 & 5,68 & 0,253 \\
Fonction de confrontation & 5,46 & 5,26 & 0,194 \\
Fonction de guide & 5,74 & 5,67 & 0,582 \\
Fonction de modèle & $\mathbf{5 , 6 7}$ & $\mathbf{5 , 1 5}$ & $\mathbf{0 , 0 0 3 * *}$ \\
\hline
\end{tabular}




\subsection{PROPOSITION D’UN MODĖLE PARCIMONIEUX}

Étant donné que les analyses précédentes incluent 35 items servant à mesurer neuf différentes sous-fonctions et fonctions, les modèles proposées ne sont pas très parcimonieux. S'il peut être intéressant dans certains contextes de pouvoir mesurer de manière précise les différentes fonctions, dans d'autres cas, il est utile d'obtenir une mesure plus simple qui mesure les différentes fonctions du mentor dans leur ensemble. Par exemple, dans le mentorat organisationnel, on a déjà proposé un tel outil (voir le travail de Pellegrini et Scandura (2005)). Pour parvenir à réduire le nombre d'items tout en conservant l'essence des différentes fonctions du mentor, l'item ayant le coefficient le plus élevé et le terme d'erreur le moins élevé de chacune des fonctions analysées précédemment a été sélectionné pour faire partie du modèle parcimonieux. De cette manière, toutes les sous-fonctions du mentor sont alors incluses et mesurées avec l'item qui les représente le mieux. Les items suivants ont donc été retenus pour l'analyse : REF1, SEC2, MOT4, CONF2, INT1, SI1, CFR4, GUI2 et MOD2 (voir Tableau 9).

Tableau 9. Outil de mesure parcimonieux des fonctions du mentor

\begin{aligned} & \multicolumn{1}{c}{ Item } \multicolumn{1}{c}{ Libellé } \\ & \hline REF1 Il me permet de me faire une image précise de moi et de mon entreprise \\ & SEC2 Il me sécurise \\ & MOT4 Il croit que je peux réussir comme entrepreneur \\ & CONF2 Je le considère comme un ami \\ & INT1 Il me met en relation avec des gens qu'il connaît \\ & SI1 Il me fournit des informations et des renseignements reliés au monde des affaires \\ & CFR4 Il n'hésiterait pas à me contredire s'il n'était pas d'accord \\ & GUI2 Il me propose d'autres points de vue \\ & MOD2 Il m'expose ses réussites et ses échecs \\ & \hline\end{aligned}

Comme on peut le voir au modèle de la Figure 1 et au Tableau 10, ces items possèdent maintenant un coefficient d'erreur plus élevé et ils sont tous significatifs à $p>0,05$. Néanmoins, tous les items sont significatifs à $p \leq 0,01$ pour expliquer la variable latente. De plus, le $\chi^{2}$ est $36,29$ pour 27 degrés de liberté ( $p=0,10908)$, le RMSEA est à 0,04667 , le SRMR est à 0,03780 et le CFI est à 0,9959 , ce qui indique un excellent modèle. Le coefficient $\alpha$ pour les neuf items est de 0,898 , ce qui dépasse aussi les seuils acceptables.

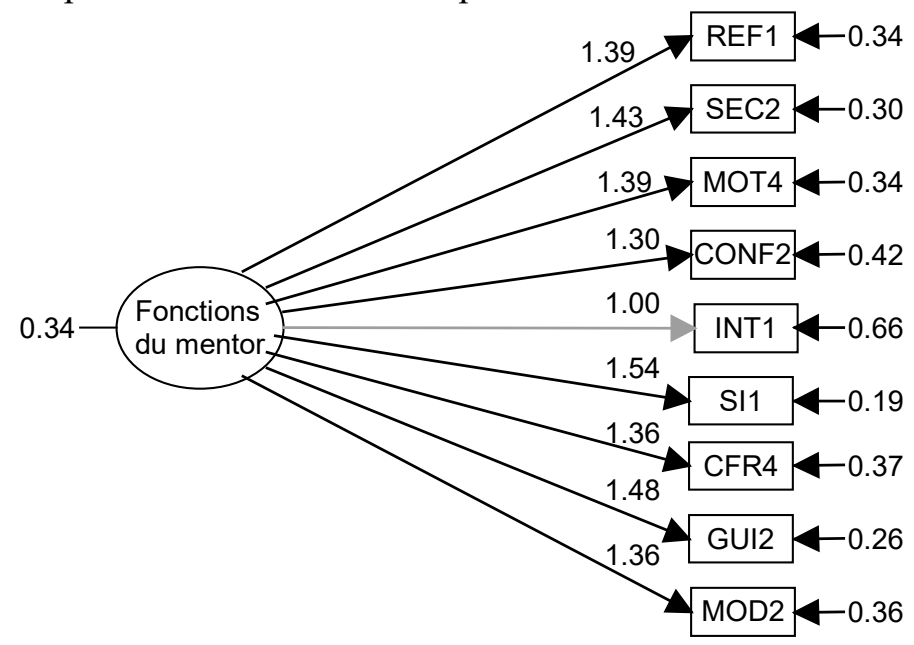

Figure 1. Modèle parcimonieux des fonctions du mentor 
Tableau 10. Estimés des paramètres du modèle parcimonieux des fonctions du mentor.

\begin{tabular}{cccc}
\hline Variable X & Variable Y & X vers Y $\left(R^{2}\right)$ & $\begin{array}{c}\text { Coef. d'erreur } \\
(\text { Sig. }(p))\end{array}$ \\
\hline Fonctions & REF1 & 0,6596 & $0,0005^{* * *}$ \\
Fonctions & SEC2 & 0,6954 & $0,0016^{* *}$ \\
Fonctions & MOT4 & 0,6567 & $0,0025^{* *}$ \\
Fonctions & CONF2 & 0,5804 & $0,0000^{* * *}$ \\
Fonctions & INT1 & 0,3422 & $0,0000^{* * *}$ \\
Fonctions & SI1 & 0,8105 & $0,0493^{*}$ \\
Fonctions & CFR4 & 0,6300 & $0,0003^{* * *}$ \\
Fonctions & GUI2 & 0,7442 & $0,0080^{* *}$ \\
Fonctions & MOD2 & 0,6366 & $0,0003^{* * *}$ \\
\hline$* p \leq 0,05 * * p \leq 0,01 * * * p \leq 0,001$ &
\end{tabular}

Bref, les résultats de l'analyse indiquent que les neuf items sélectionnés sont adéquats pour représenter un modèle parcimonieux des fonctions du mentor de l'entrepreneur.

\section{DISCUSSION ET CONCLUSION}

L'analyse précédente a permis de démontrer la justesse des construits théoriques proposés dans la partie inductive de cette recherche, à savoir que le mentor de l'entrepreneur exerce neuf différentes fonctions. Quatre de ces fonctions se regroupent pour former les fonctions psychologiques et quatre autres composent les fonctions reliées à la carrière, la dernière étant la fonction de modèle de rôle. Bien qu'il puisse être difficile parfois de statuer définitivement sur la validité d'un nouvel outil proposé, les résultats obtenus laissent penser que les fonctions du mentor possèdent une validité scientifique suffisante. Si la nature de ces neuf fonctions prend sa source dans l'analyse inductive des groupes de discussion, les regroupements ont été effectués en s'inspirant d'abord des travaux de Kram (1985), qui proposait un regroupement de fonctions psychologiques ainsi qu'un regroupement de fonctions reliées à la carrière, mais aussi des travaux de ses successeurs, qui ont démontré empiriquement que la fonction de modèle de rôle était distincte des deux précédentes (Scandura, 1992; Scandura et Ragins, 1993; Pellegrini et Scandura, 2005). Nous savons maintenant que ces regroupements sont adéquats et pertinents à d'autres contextes, dont celui du mentorat de l'entrepreneur.

L'exploration des fonctions du mentor de l'entrepreneur a déjà été faite par le passé (Waters et al., 2000) mais leur analyse n'était certainement pas complète. D'abord, le programme étudié par les auteurs précédemment cités ressemble davantage à du coaching qu'à du mentorat et faisait appel à des spécialistes (les «mentors») qui avaient pour mandat de superviser l'implantation du plan d'affaires chez le nouvel entrepreneur, en lui prodiguant des conseils au niveau marketing, finance ou en droit des affaires. Ensuite, les écrits sur le mentorat organisationnel font état parfois de trois fonctions (par exemple Scandura et Williams (2001)), dont l'une d'elles concerne le rôle de «modèle». Malgré la possibilité "théorique » que le mentor entrepreneur joue un rôle de modèle chez le jeune entrepreneur, l'outil développé par Waters et al. ignore totalement cette facette importante des fonctions du mentor. Les résultats obtenus ici par l'analyse des fonctions du mentor confirment l'importance d'inclure la fonction de modèle de rôle, ce qui démontre les limites de l'outil proposé par Waters et al. (2000) et la pertinence du nouvel outil proposé. Il a aussi été permis de constater que les items possédant les meilleurs résultats empiriques pouvaient très bien représenter un modèle parcimonieux des fonctions du mentor, lequel pourrait être utile pour mesurer le niveau global de fonctions assumées par le mentor et reçues par le mentoré, par exemple. Bien entendu, en retranchant 
plusieurs items des modèles initiaux, chaque item retenu mesure alors moins bien la fonction globale du mentor. Cependant, dans la perspective où un modèle parcimonieux pourrait s'avérer utile ou nécessaire, il s'agit d'une proposition qui est tout à fait acceptable.

Concernant la généralisation à d'autres programmes de mentorat, il est permis de penser que l'outil proposé serait adaptable à différents contextes, et pas seulement à celui du réseau de mentorat de la Fondation de l'entrepreneurship. D'abord, il faut garder à l'esprit le caractère décentralisé de l'organisation des services de mentorat du côté de la Fondation. Les coordonnateurs ont une grande liberté dans le choix des mentors, le choix des dyades, et ainsi de suite. Si certains coordonnateurs partagent l'avis des dirigeants du réseau à l'effet que le mentor ne doit pas donner de conseils et ne doit pas être un spécialiste d'une discipline de gestion, d'autres coordonnateurs procèdent à des jumelages où le mentor est un spécialiste d'une discipline et où le mentoré possède une faiblesse et souhaite s'améliorer. Cette décentralisation dans la gestion amène une plus grande diversité des directives transmises aux mentors et une pratique moins restrictive que si les coordonnateurs étaient à l'emploi de la Fondation. Sachant que dans l'ensemble du réseau, il existe une diversité de mentors et de types d'intervention, l'étendue des fonctions du mentor risque d'être davantage diversifiée. Conséquemment, la nature des fonctions proposées n'est pas implicitement liée au contexte du programme de mentorat. Par exemple, au niveau des fonctions reliées à la carrière, les items ne font pas référence à la nature précise de l'intervention. Dans aucun des libellés il n'est question d'aide au niveau du marketing, de la finance, ou autre. Il est plutôt question, par exemple, d'une fonction de "guide », qui suggère de nouvelles options, propose d'autres points de vue, donne des conseils à propos des problèmes vécus et aide à clarifier le problème. Cette fonction pourrait s'appliquer à différents contextes, même auprès de cadres dans la grande organisation. C'est aussi le cas pour toutes les autres fonctions reliées à la carrière, mais aussi pour celles de nature psychologique. Bref, même si l'outil prend sa source dans le réseau de mentorat de la Fondation, sa généralisation à d'autres dispositifs existants ne semble pas menacée pour autant, mais mérite néanmoins d'être vérifiée.

Bien que l'analyse de réseau nomologique n'ait été effectuée que partiellement, notamment en utilisant une méthode similaire à celle du «test-retest» et en vérifiant la relation entre la fonction d'intégration et le nombre de personnes présentées par le mentor, il est possible que l'outil puisse être influencé par d'autres concepts similaires. En effet, on a constaté que le nombre de personnes présentées par le mentor était corrélé significativement aussi aux autres fonctions reliées à la carrière, même si la corrélation était moins grande que pour la fonction d'intégration. Cela souligne d'ailleurs le rôle du mentor en tant qu'intermédiaire facilitant l'intégration du mentoré dans la communauté d'affaires. Cette situation suggère d'autres analyses spécifiques visant à prouver le réseau nomologique des concepts proposés, lesquelles ouvrent la porte aux recherches futures. Par contre, il faut souligner que le concept des fonctions du mentor a retenu beaucoup l'attention des chercheurs dans le contexte du mentorat dans la grande organisation et que, conséquemment, la possibilité que ces construits correspondent à une certaine réalité observable dans un contexte de mentorat d'entrepreneur s'en trouve renforcée. En outre, il faut rappeler que la fonction de modèle de rôle est significativement différente selon que le mentor ait été un entrepreneur ou qu'il ait eu une autre carrière. Ces résultats donnent du crédit à l'analyse du réseau nomologique, même si elle n'est que partielle, et laissent suggérer que les fonctions du mentor soient tout à fait valides. Il convient de souligner aussi que même si la plupart des coefficients d'erreurs des construits sont non significatifs, certains le sont et indiquent certaines faiblesses dans la formulation des items, par exemple une imprécision des concepts mesurés. Bien entendu, ces faiblesses demeurent mineures puisque les résultats obtenus par les indices d'ajustement démontrent la qualité du construit dans son ensemble. Néanmoins, pour les recherches futures, ces pistes pour des ajustements à l'outil pourraient être prises en considération et mener à un raffinement des mesures des fonctions du mentor. De façon particulière, de nouveaux items pourraient être 
proposés pour un modèle parcimonieux des fonctions du mentor. En effet, ceux utilisés pour l'analyse ont été développés dans une perspective «multi-items » et pourraient sans doute être améliorés dans leur formulation.

Malgré ces limites, les analyses ont permis de faire avancer les connaissances sur cet objet de recherche. Les résultats permettent notamment l'utilisation de l'outil de mesure développé pour considérer le rôle de certaines variables psychologiques du mentoré dans le déploiement des fonctions de son mentor, d'une part, et l'impact des fonctions du mentor dans le développement de certaines retombées chez le mentoré, d'autre part. Ces analyses constituent des pistes pour les recherches futures. Également, la «boîte noire » du mentorat pour entrepreneur est maintenant mieux comprise, ce qui permet de démystifier cette forme d'accompagnement qui gagne en popularité. Les dispositifs souhaitant mettre en place une telle forme de soutien pourront former des mentors conscients des différentes fonctions qu'ils pourraient être appelés à exercer auprès d'un entrepreneur novice. Bien entendu, tout repose sur les besoins initiaux des entrepreneurs : quelle forme d'aide est-elle recherchée ? En sachant l'éventail des fonctions du mentor, ceux-ci pourront mieux juger si l'aide apportée correspond à leurs attentes. Ce ne sont là que quelques utilités du travail présenté ici, qui se veut un premier pas dans la compréhension du phénomène d'intérêt.

\section{RÉFÉRENCES}

ALLEN, T.D. et EBY, L.T. (2004), "Factors Related to Mentor Reports of Mentoring Functions Provided: Gender and Relational Characteristics", Sex Roles, Vol. 50, nº1/2, p.129-139.

ARENIUS, P. et MINNITI, M. (2005), "Perceptual Variables and Nascent Entrepreneurship", Small Business Economics, Vol. 24, n³, p.233-247.

ARMSTRONG, J.S. et OVERTON, T.S. (1977), "Estimating Nonresponse Bias in Mail Surveys", Journal of Marketing Research, Vol. 14, n³, p.396-402.

BARON, R.A. et ENSLEY, M.D. (2006), "Opportunity Recognition as the Detection of Meaningful Patterns: Evidence from Comparisons of Novice and Experienced Entrepreneurs", Management Science, Vol. 52, n9, p.1331-1344.

BISK, L. (2002), "Formal entrepreneurial mentoring: the efficacy of third party managed programs", Career Development International, Vol. 7, nº5, p.262-270.

BOUQUILLON, E.A., SOSIK, J.J. et LEE, D. (2005), "'It's only a phase': examining trust, identification and mentoring functions received accross the mentoring phases", Mentoring \& Tutoring, Vol. 13, n², p.239-258.

COHEN, N.H. (1995), Mentoring Adult Learners - A Guide for Educators and Trainers, Krieger Publishing Company.

COUTERET, P., ST-JEAN, E. et AUDET, J. (2006), "Le mentorat : conditions de réussite de ce mode d'accompagnement de l'entrepreneur", 23e conférence du CCPME/CCSBE, TroisRivières, Québec, 28-30 septembre.

D'ABATE, C.P., EDDY, E.R. et TANNENBAUM, S.I. (2003), "What's in a Name? A Literature-Based Approach to Understanding Mentoring, Coaching, and Other Constructs That Describe Developmental Interactions", Human Resource Development Review, Vol. 2, nº 4, p.360-384.

DIOCHON, M., GASSE, Y., MENZIES, T.V. et GARAND, D. (2001), "From conception to inception: Initial findings from the Canadian study on entrepreneurial emergence", Administrative Sciences Association of Canada, London (Ontario), 27-29 mai.

DRUCKER-GODARD, C., EHLINGER, S. et GRENIER, C. (2003), "Validité et fiabilité de la recherche", dans Méthodes de recherche en management, Ed. par R.-A. THIÉTART (2 éd.). Dunod, p.257-287.

FORTIN, J. et SIMARD, P. (2007), "La sagesse au profit des PME : caractéristiques et rôles du mentor d'entrepreneurs", Journal of Small Business and Entrepreneurship, Vol. 20, nº2, p.201-215. 
FOWLER, J. (2002), Mentoring relationships at work: An investigation of mentoring functions, benefits, and gender. Doctoral Thesis, Griffith University, Nathan, Australia

GASSE, Y. (2003). Impact of the community on business creation, Document de travail $\mathrm{n}^{-}$ 2003-019, Université Laval, Québec.

GERBING, D.W. et ANDERSON, J.C. (1988), "An updated paradigm for scale development incorporating unidimensionality and its assessment", Journal of Marketing Research, Vol. 25, n², p.186-192.

HANSFORD, B., TENNENT, L. et EHRICH, L.C. (2002), "Business Mentoring: Help or Hindrance?", Mentoring \& Tutoring, Vol. 10, n², p.101-115.

HATTIE, J. (1985), "Methodology Review: Assessing Unidimensionality of Tests and Items", Applied Psychological Measurement, Vol. 9, n², p.139-164.

HINKIN, T.R. (1998), "A Brief Tutorial on the Development of Measures for Use in Survey Questionnaires", Organizational Research Methods, Vol. 1, nº1, p.104-121.

HOUDE, R. (1995), Des mentors pour la relève, Éditions du Méridien.

JACOBI, M. (1991), "Mentoring and Undergraduate Academic Success: A Literature Review", Review of Educational Research, Vol. 61, nº 4, p.505-532.

JÖRESKOG, K. et SÖRBOM, D. (2002), PRELIS 2: User's Reference Guide, (3e éd.), SSI inc.

KRAM, K.E. (1985), Mentoring at Work : Developmental Relationships in Organizational Life Scott Foresman.

LEVESQUE, L.L., O'NEILL, R.M., NELSON, T. et DUMAS, C. (2005), "Sex differences in the perceived importance of mentoring functions", Career Development International, Vol. $10, n^{\circ} 6 / 7$, p.429-444.

MCCAULEY, C.D. et DOUGLAS, C.A. (2004), "Developmental Relationships", dans The Center for Creative Leadership Handbook of Leadership Development, Ed. par C. D. MCCAULEY et E. VAN VELSOR (2e éd.). Jossey-Bass, p.85-115.

MCGREGOR, J. et TWEED, D. (2002), "Profiling a New Generation of Female Small Business Owners in New Zealand: Networking, Mentoring and Growth", Gender, Work and Organization, Vol. 9, n⿳⺈, p.420-438.

NANDRAM, S.S. (2003), "Entrepreneurs' Need For Mentoring And Their Individual Differences", ICSB $48^{\text {th }}$ World Conference, International Council for Small Business, North Ireland, June, 15-18.

NOE, R.A. (1988), "An Investigation of the determinants of successful assigned mentoring relationships", Personnel Psychology, Vol. 41, n³, p.457-479.

PELLEGRINI, E.K. et SCANDURA, T.A. (2005), "Construct equivalence across groups: an unexplored issue in mentoring research", Educational and Psychological Measurement, Vol. $65, \mathrm{n}^{\circ} 2$, p.323-335.

ROCK, A. et GARAVAN, T. (2006), "Reconceptualizing developmental relationships", Human Resource Development Review, Vol. 5, n³, p.330-354.

RUSH, B.L., GRAHAM, J.B. et LONG, W.A. (1987), "The Use of Peer Networks In The StartUp Process", Frontiers of Entrepreneurship Research, Babson College, Babson, MA.

SCANDURA, T.A. (1992), "Mentorship and Career Mobility: An Empirical Investigation", Journal of Organizational Behavior, Vol. 13, n², p.169-174.

SCANDURA, T.A. et RAGINS, B.R. (1993), "The Effects of Sex and Gender Role Orientation on Mentorship in Male-Dominated Occupations", Journal of Vocational Behavior, Vol. $43, \mathrm{n}^{\circ} 3, \mathrm{p} .251-265$.

SCANDURA, T.A. et WILLIAMS, E.A. (2001), "An Investigation of the Moderating Effects of Gender on the Relationships between Mentorship Initiation and Protégé Perceptions of Mentoring Functions", Journal of Vocational Behavior, Vol. 59, n³, p.342-363.

SHEPHERD, D.A. et KRUEGER, N.F.J. (2002), "An Intentions-Based Model of Entrepreneurial Teams' Social Cognition", Entrepreneurship: Theory \& Practice, Vol. $27, n^{\circ} 2$, p.167-185. 
SHOOK, C.L., KETCHEN, D.J.J., HULT, T.M. et KACMAR, K.M. (2004), "An assessment of the use of structural equation modeing in strategic management research", Strategic Management Journal, Vol. 25, nº 4, p.397-404.

SLATER, S.F. et ATUAHENE-GIMA, K. (2004), "Conducting survey research in strategic management", dans Research Methodology in Strategy and Management, Ed. par D. J. J. KETCHEN et D. D. BERGH. Elsevier, p.227-250.

ST-JEAN, E. (2008), "La formation destinée à l'entrepreneur novice : exploration des possibilités offertes par le mentorat", Revue de l'Entrepreneuriat, Vol. 7, $\mathrm{n}^{\circ} 1, \mathrm{p} .1-22$.

TABACHNICK, B.G. et FIDELL, L.S. (2007), Using Multivariate Statistics, (5e éd.), Allyn \& Bacon.

TEPPER, K., SHAFFER, B.C. et TEPPER, B.J. (1996), "Latent structure of mentoring function scales", Educational and Psychological Measurement, Vol. 56, n5, p.848-857.

WANBERG, C.R., WELSH, E.T. et HEZLETT, S.A. (2003), "Mentoring Research: A Review and Dynamic Process Model", dans Research in Personnel and Human Resources Management, Ed. par J. J. MARTOCCHIO et G. R. FERRIS. Elsevier Science Ltd., p.39-124.

WATERS, L., MCCABE, M., KIELlERUP, D. et KIELLERUP, S. (2000). A brief scale to measure the role of mentoring in small business start-up, Working Paper $\mathrm{n}^{\mathrm{0}} 14$, The University of Melbourne, Departement of Management, Melbourne, $18 \mathrm{p}$.

WIKHOLM, J., HENNINGSON, T. et HULTMAN, C.M. (2005), "Demand of mentoring among new starters", ICSB $50^{\text {th }}$ World Conference, International Council for Small Business, Washington, DC. 